\title{
Interpolation by means of Given Differences (Central-Difference Notation).
}

\author{
By W. F. Sheppard \\ (Received 17th March, 1931. Read 4th December, 1931.)
}

1. Formulae of interpolation in terms of given central differences might be regarded as falling into two groups, $A$ and $B$. In group $A$, the simplest cases are those in which each given difference is one of the two which in the difference table lie nearest to the preceding given difference; the differences are all natural differences (i.e., are not mean differences), and are all expressed in the centraldifference notation. Any such formula can be a central-difference formula for a certain range of the variable: but that is a matter with which we are only incidentally concerned. What I have to do is to examine the formula as determined by the series of differences given. I have then to see how the formula is affected when an ordinary difference is replaced by a mean difference. This brings us to group B, which comprises two formulae only: the Newton-Stirling formula, which expresses the required quantity in terms of a tabulated value and its central differences; and the Newton-Bessel formula, which expresses it in terms of the mean of two tabulated values and the central differences of this mean.

The related quantities are denoted by $x$ and $u$ : the values of $x$ proceed by intervals $h$; and $u_{\theta}$ is an expression for the value of $u$ corresponding to value $x_{\theta} \equiv x_{0}+\theta h$ of $x$. Also $(n, r)$ means

$$
\frac{n(n-1) \ldots(n-r+1)}{r !} \text {. }
$$

For simplicity of phrasing, the initial $u$ of the formula will be counted as a "difference."

2. I will begin with a Group A formula in the advancingdifference notation. Suppose that our data are the values of

$$
u_{7}, \Delta u_{7}, \Delta^{2} u_{7}, \Delta^{3} u_{6}, \Delta^{4} u_{6}, \Delta^{5} u_{6} .
$$

Then the formula for $u_{\theta}$ is (cf. Whittaker and Robinson, ref. 5, p. 46)

$$
\begin{aligned}
u_{\theta}=u_{7} & +(\theta-7,1) \Delta u_{7}+(\theta-7,2) \Delta^{2} u_{7}+(\theta-7,3) \Delta^{3} u_{6} \\
& +(\theta-6,4) \Delta^{4} u_{6}+(\theta-6,5) \Delta^{5} u_{6} .
\end{aligned}
$$

The rule for construction of successive terms of this expression is obvious: the coefficient in the term which follows any given difference 
$\Delta^{p} u_{n}$ is $(\theta-n, p+1)$. This rule is, in fact, nothing more than a summary of the rule given by Mr D. C. Fraser for the use of his diagram in its simpler form (ref. 2, $\$ 10$, pp. 240-241).

(It may be noted that the formula is a central-difference interpolation formula if $\theta$ lies between 8 and 9.)

This relation between the difference occurring in a term of the formula and the coefficient in the following term is referred to by Dr Aitken in a recent paper on generalisation of interpolation formulae (ref. 1, p. 110). From the purely formal point of view, we could divide up $u_{\theta}$ into linked segments by dividing each term into coefficient and difference:-

$$
\begin{aligned}
u_{\theta}= & \left|u_{7}+(\theta-7,1)\right| \Delta u_{\tau}+(\theta-7,2)\left|\Delta^{2} u_{7}+(\theta-7,3)\right| \\
& \left|\Delta^{3} u_{6}+(\theta-6,4)\right| \Delta^{4} u_{6}+(\theta-6,5) \mid \Delta^{5} u_{6}+\ldots
\end{aligned}
$$

3. Now let us express (I) in terms of central differences. It becomes

$$
\begin{aligned}
u_{\theta}=u_{7} & +(\theta-7,1) \delta u_{7 \frac{1}{1}}+(\theta-7,2) \delta^{2} u_{8}+(\theta-7,3) \delta^{3} u_{i^{\frac{1}{3}}} \\
& +(\theta-6,4) \delta^{4} u_{8}+(\theta-6,5) \delta^{5} u_{8 \frac{1}{2}} \ldots
\end{aligned}
$$

No simple rule for determining the coefficients in this expression suggests itself. The reason is that the notation of the factorial coefficients $(\theta-7,1)$, etc., is not consistent with the system of notation of central differences: we require a notation which shall be consistent with this system.

4. The difficulty is to find a notation that will please everybody. In the ordinary notation of factorials, $n(n-1)$ is denoted by $(n, 2)$, and is thus regarded as being a function of $n$. But in constructing a notation which shall fit in with the use of central differences we must adopt the principles of the central-difference system. On these principles, $\frac{1}{2} n(n-1)$ must be regarded as a function of $n-\frac{1}{2}$. Similarly $\frac{1}{6} n(n-1)(n-2)$ must be regarded as a function of $n-1$; and so on.

In writing a paper on this subject some years ago (ref. 3, p. 81), I had to adopt some notation. Bearing in mind the common symbol, occurring in the expansion of $(1+x)^{n}$,

$$
(n, p) \equiv \frac{n(n-1) \ldots(n-p+1)}{p !},
$$

and the less common symbol, occurring in the expansion of $(1-x)^{-n}$,

$$
[n, p] \equiv \frac{n(n+1) \ldots(n+p-1)}{p !},
$$


I took a middle course, and wrote

$$
\begin{aligned}
(n, q] & \equiv \frac{\left(n-\frac{1}{2} q+\frac{1}{2}\right)\left(n-\frac{1}{2} q+\frac{3}{2}\right) \ldots\left(n+\frac{1}{2} q-\frac{1}{2}\right)}{q !}, \\
{[n, s) } & \equiv \frac{1}{2}\left\{\left(n-\frac{1}{2}, s\right]+\left(n+\frac{1}{2}, s\right]\right\} \\
& =\frac{n}{s}(n, s-1],
\end{aligned}
$$

where $q$ and $s$ are positive integers, and $n$ is any number, positive or negative. I do not know whether this notation has been adopted at all. I may mention that Dr J. F. Steffensen (ref. 4, pp. 8, 9) adopts a different notation, but it does not seem quite satisfactory for our present purpose.

With my notation, (II) becomes

$$
\begin{aligned}
u_{\theta}=u_{7} & +(\theta-7,1] \delta u_{7 \frac{1}{2}}+\left(\theta-7 \frac{1}{\frac{1}{2}}, 2\right] \delta^{2} u_{8}+(\theta-8,3] \delta^{3} u_{\overline{7} \frac{1}{2}} \\
& +\left(\theta-7 \frac{1}{2}, 4\right] \delta^{1} u_{8}+(\theta-8,5] \delta^{5} u_{8 \frac{1}{3}} .
\end{aligned}
$$

The rule for successive terms now becomes clear: it is that the coefficient in the term which follows any given difference $\delta^{i} u_{n}$ is $(\theta-n, q+1]$.

The expression can be divided into segments in a manner analogous to (I.A).

5. Next take the case in which the differences to be used include a mean difference and the natural differences on the two sides of it. 'In the above example, in which $\delta^{2} u_{8}, \delta^{3} u_{\tau_{3}}$, and $\delta^{4} u_{\varsigma}$ are given differences, suppose that instead of being given $\delta^{3} u_{7_{2}}$ we are given $\mu \delta^{3} u_{8}$. We want to find $u_{\theta}$ in terms of the given differences.

The relevant part of the table of differences is given below.

$$
\begin{array}{ccc}
\left(\mu \delta^{2} u_{7 \frac{1}{2}}\right) & \delta^{3} u_{7 \frac{1}{2}} & \left(\mu \delta^{4} u_{7 \frac{7}{2}}\right) \\
\delta^{2} u_{8} & \left(\mu \delta^{3} u_{8}\right) & \delta^{4} u_{8} \\
\left(\mu \delta^{2} u_{\delta \frac{1}{2}}\right) & \delta^{3} u_{\delta_{\frac{1}{2}}} & \left(\mu \delta^{4} u_{\delta_{\frac{1}{2}}}\right)
\end{array}
$$

It will be seen that instead of the pair of diagonal steps $\delta^{2} u_{8}-\delta^{2} u_{7 \frac{1}{2}}-\delta^{4} u_{8}$ we have a pair of horizontal steps $\delta^{2} u_{8}-\mu \delta^{3} u_{8}$ - $\delta^{4} u_{3}$. The expression for $u_{\theta}$ will obviously be the mean of two expressions, which respectively use

and

$$
\begin{array}{llllll}
u_{7} & \delta u_{7^{\frac{1}{2}}} & \delta^{2} u_{8} & \delta^{3} u_{i^{\frac{1}{2}}} & \delta^{4} u_{8} & \delta^{5} u_{3 \frac{1}{2}}
\end{array}
$$

$$
\begin{array}{lllllll}
u_{7} & \delta u_{7 \frac{1}{2}} & \delta^{2} u_{8} & \delta^{3} u_{8 \frac{1}{2}} & \delta^{4} u_{8} & \delta^{5} u_{8 \underline{\underline{1}}}
\end{array}
$$


By the rule stated in $\S 4$, the first three terms and the sixth term are alike in the two expressions; these are given in (V). The fourth and fifth terms are respectively

and

$$
\ldots+(\theta-8,3] \delta^{3} u_{7 \frac{1}{2}}+\left(\theta-7 \frac{1}{2}, 4\right] \delta^{4} u_{8}+\ldots
$$

Taking the mean, we get a formula which differs in its fourth and fifth terms from (V): this formula is, by (IV),

$$
\begin{aligned}
u_{\theta}=u_{7} & +(\theta-7,1] \delta u_{7 \frac{1}{2}}+\left(\theta-7 \frac{1}{2}, 2\right] \delta^{2} u_{8}+(\theta-8,3] \mu \delta^{3} u_{8} \\
& +[\theta-8,4) \delta^{4} u_{8}+(\theta-8,5] \delta^{5} u_{8 \frac{1}{2}} \ldots
\end{aligned}
$$

Thus we get the rule: If the given differences include a mean difference $\mu \delta^{a} u_{n}$ and the natural differences on the two sides of it (namely $\delta^{s-1} u_{n}$ and $\left.\delta^{s+1} u_{n}\right)$, the rule of $\$ 4$ applies to all the terms, except that in the term following the mean difference the coefficient is $[\theta-n, s+1)$ instead of $(\theta-n, s+1]$.

The data for $u_{\theta}$ might include two or more mean differences, and the above rule would apply for each such difference: provided, of course, that the adjoining differences on the two sides of it are natural differences.

The above requires obvious modification for the case in which $u_{\theta}$ begins with a mean difference.

6. The extreme cases are those in which natural and mean differences occur alternately, in a horizontal line. These are the cases mentioned in $\S 1$ as constituting Group B; namely, the NewtonStirling formula and the Newton-Bessel formula. In our notation they are as follows:-

$$
\begin{aligned}
\boldsymbol{u}_{\theta}=u_{0} & +(\theta, 1] \mu \delta u_{0}+[\theta, 2) \delta^{2} u_{0}+(\theta, 3] \mu \delta^{3} u_{0}+[\theta, 4) \delta^{4} u_{0} \\
& +(\theta, 5] \mu \delta^{5} u_{0}+\ldots \\
u_{\theta}=\mu u_{\frac{1}{2}} & +\left[\theta-\frac{1}{2}, 1\right) \delta u_{\frac{1}{2}}+\left(\theta-\frac{1}{2}, 2\right] \mu \delta^{2} u_{\frac{1}{3}}+\left[\theta-\frac{1}{2}, 3\right) \delta^{3} u_{\frac{1}{2}} \\
& +\left(\theta-\frac{1}{2}, 4\right] \mu \delta^{4} u_{\frac{1}{2}}+\left[\theta-\frac{1}{2}, 5\right) \delta^{5} u_{\frac{1}{2}}+\ldots
\end{aligned}
$$

But, in view of (IV), they may also be written as follows; $\mu$ operating on $\theta$ or on $u$ according to its position in the term:-

$$
\begin{aligned}
u_{\theta}=u_{0} & +(\theta, 1] \cdot \mu \delta u_{0}+\mu(\theta, 2] \cdot \delta^{2} u_{0}+(\theta, 3] \cdot \mu \delta^{3} u_{0} \\
& +\mu(\theta, 4] \cdot \delta^{4} u_{0}+(\theta, 5] \cdot \mu \delta^{5} u_{0}+\ldots \\
u_{\theta}=\mu u_{1} & +\mu\left(\theta-\frac{1}{2}, 1\right] \cdot \delta u_{1}+\left(\theta-\frac{1}{2}, 2\right] \cdot \mu \delta^{2} u_{1}+\mu\left(\theta-\frac{1}{2}, 3\right] \cdot \delta^{3} u_{1} \\
& +\left(\theta-\frac{1}{2}, 4\right] \cdot \mu \delta^{4} u_{1}+\mu\left(\theta-\frac{1}{2}, 5\right] \cdot \delta^{5} u_{1}+\ldots \ldots
\end{aligned}
$$


The curious thing is that in each formula a $\mu$ appears in every term (including $u_{0}$ in (IX) if it is written $\mu(\theta, 0], u_{0}$ ) but acts alternately on $\theta$ and on $u$. The reason of this is the close relation (cf. $\S 2$ ) between the difference in any term and the coefficient in the next term; this relation is rather obscured by the fact that in each of the two formulae the suffix to $u$ is the same throughout. The points in each formula, like the lines in (I.A), divide the formula into linked segments, each of which has either no $\mu$ or two $\mu$ 's.

7. The above seems to be about as far as we can go in our search for simple general formulas: but Dr Aitken (ref. 1, 110-112) has indicated a method for expressing $u_{\theta}$ in terms of any sufficient set of natural differences, however distant they are from one another in the difference-table; and his examples suggest that the method can be extended to cases in which the data include any number of mean differences, without restriction as to position.

\section{REFERENCES.}

1. A. C. Aitken, "On a generalisation of formulae for polynomial interpolation," Journal of the Institute of Actuaries, 61 (1930), 107-112.

2. D. C. Fraser, "On the graphic delineation of interpolation formulae," Journal of the Institute of Actuaries, 43 (1909), 235.241.

3. W. F. Sheppard, "Factorial moments in terms of sums or differences," Pruceedings of the London Mathematical Society, series 2, 13 (1913), 81-96.

4. J. F. Steffensen, "Interpolation" (1927).

5. E. T. Whittaker and G. Robinson, "The Calculus of Observations" (1924). 